\title{
Development of a Control Banding Tool for Nanomaterials
}

\author{
M. Riediker, ${ }^{1}$ C. Ostiguy, ${ }^{2}$ J. Triolet, ${ }^{3}$ P. Troisfontaine, ${ }^{4}$ D. Vernez, ${ }^{1}$ G. Bourdel, ${ }^{5}$ \\ N. Thieriet, ${ }^{5}$ and A. Cadène ${ }^{5}$ \\ ${ }^{1}$ Institute for Work and Health-IST [Institut Universitaire Romand de Santé au Travail], Rue du Bugnon 21, \\ 1011 Lausanne, Switzerland \\ ${ }^{2}$ Institut de Recherche Robert-Sauvé en Santé et en Sécurité du Travail-IRSST, Montréal, Canada H3A 3C2 \\ ${ }^{3}$ Institut National de Recherche et de Sécurité-INRS, 30 rue Olivier Noyer, 75680 Paris, France \\ ${ }^{4}$ Institut Scientifique de Santé Publique, Rue Juliette Wytsmanstraat 14, 1050 Brussels, Belgium \\ ${ }^{5}$ French Agency for Food, Environmental and Occupational Health Safety ANSES, 27-31 Avenue du Général Leclerc, \\ 94701 Maisons-Alfort, France
}

Correspondence should be addressed to M. Riediker, michael.riediker@alumni.ethz.ch

Received 21 January 2012; Accepted 19 April 2012

Academic Editor: Paul A. Schulte

Copyright (c) 2012 M. Riediker et al. This is an open access article distributed under the Creative Commons Attribution License, which permits unrestricted use, distribution, and reproduction in any medium, provided the original work is properly cited.

Control banding (CB) can be a useful tool for managing the potential risks of nanomaterials. The here proposed $\mathrm{CB}$, which should be part of an overall risk control strategy, groups materials by hazard and emission potential. The resulting decision matrix proposes control bands adapted to the risk potential levels and helps define an action plan. If this plan is not practical and financially feasible, a full risk assessment is launched. The hazard banding combines key concepts of nanomaterial toxicology: translocation across biological barriers, fibrous nature, solubility, and reactivity. Already existing classifications specific to the nanomaterial can be used "as is." Otherwise, the toxicity of bulk or analogous substances gives an initial hazard band, which is increased if the substance is not easily soluble or if it has a higher reactivity than the substance. The emission potential bands are defined by the nanomaterials' physical form and process characteristics. Quantities, frequencies, and existing control measures are taken into account during the definition of the action plan. Control strategies range from room ventilation to full containment with expert advice. This CB approach, once validated, can be easily embedded in risk management systems. It allows integrating new toxicity data and needs no exposure data.

\section{Introduction}

Nanomaterials are materials with external structures in the nanoscale, namely, between 1 and $100 \mathrm{~nm}$ [1]. They often exhibit properties that differ from those of the same material which do not have nanoscale features, which provides opportunities for novel applications [2]. However, there are also many uncertainties and questions related to potentially new risks to workers and environment [3-5], in particular the identification and quantitative assessment of hazards and exposure to nanomaterials. The current occupational exposure limits (OELs) are mostly defined for dusts which are in the micrometer range at the workplace (e.g., inhalable or respirable dusts). OELs exist for inert dust and for specific dust-bound substances [6-9]. However, these values are not necessarily appropriate when applied to manufactured nanomaterials (MNM).
Occupational risk management aims at establishing the appropriate means and techniques to ensure the safety and health of employees. For chemical risks, the combination of exposure and hazard of a chemical enables a quantitative assessment of the risks. Identifying hazards involves an exhaustive inventory of all chemicals found in an establishment, followed by the compilation of accurate, detailed information on their potential hazards, mainly from labels and safety data sheets. Estimating exposure requires a study of the processes and procedures implemented, the quantities handled, the duration and frequency of operations, the properties of the chemical, and so forth [10].

In the particular case of MNM, risk assessment is difficult due to the many uncertainties related to both the identification of potential hazards and the characterization of exposure [11]. Given the current state of knowledge on 
MNM, it is highly likely that many years will be needed before we precisely know which types of nanomaterials and associated doses represent a real danger to humans and their environment. This makes quantitative risk assessment almost impossible for most work situations. In this context, the "Control Banding" method was presented as an alternative solution $[12,13]$.

Control Banding (CB) is an established method to conduct a qualitative risk assessment and to take measures to protect workers. It was developed in the pharmaceutical industry to ensure the safety of workers applying processes using products for which little information was available. These new products were allocated to "bands," mainly defined according to the hazard level of known products similar to those used, taking into account the assessment of exposure at the work place. Each band corresponded to a risk control strategy [14]. Shortly after, the UK Health and Safety Executive developed a banding method called COSHH Essentials [15-18], that was easier to use and more accessible for small professional organisations that could not afford the expertise of an occupational hygienist. A similar scheme to COSHH Essentials was described in a practical guide published by the "German Federal Institute for Occupational Safety and Health" [19]. The Stoffenmanager tool [17] proposes a further development by combining a hazard allocation scheme similar to COSHH Essentials and an exposure band allocation method based on a simplified exposure model that is easy for nonexperts to understand and use. Exposure estimates for nanomaterials are currently associated with considerable uncertainties [20] and pose serious challenges even to experts. The here proposed approach therefore relies initially only on the emission potential of the nanomaterials' physical form and on processes characteristics.

Although $\mathrm{CB}$ is a potential solution, there are few useable models adapted for use in activities related to the research, production, or processing of MNM. One conceptual approach incorporated the same control schemes as the British HSE tool [2]. Other, more recent tools [12, 21] attempted to take account of current knowledge on the toxicology of nanomaterials applied to the above-described $\mathrm{CB}$ structure.

The aim of the here presented study was the development of an operational CB approach for small to large enterprises using a hazard classification that is based on few, very fundamental physicochemical and toxicological properties of nanomaterials. It was already published in the form of a report [22]. It is designed to allow taking into account existing hazard data and the process the material will endure to assess an emission potential, and it enables an easy integration of new data expected to be generated over the coming years. Classifying MNMs in these hazard bands will ultimately provide producers and users of these substances with input data for risk management according to control levels, or "Control Banding".

\section{The Proposed Control Banding Approach}

2.1. Integration into the Health and Safety Management at Work. The control banding tool is an integral part of the overall system of health and safety management at work established by the employer based on the PDCA (Plan, Do, Check, Act) model [23]. It requires input data, irrespective of the phase of the MNM's life cycle, such as information collected at the work place through observation of actual work, toxicology data as far as available, material and matrix properties, and process characteristics. The proposed CB approach consists of the steps planning, implementation, and check and correction. Though this paper focuses mostly on the planning step, the following steps are of equal importance for the success of the $\mathrm{CB}$. The output generated by the $\mathrm{CB}$ will have an impact on other processes of the overall management system defined by the employer. The tool assumes that there is a "central support" that overlooks the risk management strategy and that provides risk assessment expertise. This can be either a health and safety office in the case of a large company where the production line units apply the $\mathrm{CB}$, or it can be a risk consultant (private or government-based, depending on the company- and country-specific situation) that supports a series of SMEs in their risk management. It is the role of this overarching management system to ensure that the CB is included in the PDCA cycle and that in addition there is a scheduled repeated review of the input parameters used for the $\mathrm{CB}$ approach as well as a review and update of the measures considered to be appropriate for the required control bands.

The planning step of the CB-tool (Figure 1) is central with regard to nanomaterials because it allocates the nanomaterial or a product containing MNM to hazard and emission potential bands and defines the feasibility and programme of the action plan for a given period. If the hazard is estimated as "very high" or if it cannot be estimated, a full hazard assessment is needed. The combination of hazard and emission potential band leads to a possible $\mathrm{CB}$ strategy. The practical and financial feasibility of this strategy is then evaluated. If it is feasible, an action plan will be defined. Otherwise, a full risk assessment is needed in lieu of the CB-approach. "Full risk assessment" means that an expert, for example an occupational hygienist, assesses the risk and proposes a risk management for the specific case by including information about hazard, emission potential, exposure, quantities used, process characteristics, existing or proposed controls, and other activity-specific information. "Full hazard assessment" means that the person applying the $\mathrm{CB}$ requests a banding decision from a toxicologist through the central support.

2.2. Allocating Hazard Bands. Hazard bands are levels of similar severity of a material's hazard. They relate to various criteria for toxicity, described or suspected, in the literature or technical documentation (labelling, product classification). For the allocation of an MNM to a hazard band, we consider only the potential hazard of the present MNM, whether raw or incorporated in a matrix (liquid or solid). Figure 2 shows the decision diagram; the reasoning is discussed later in more detail. First, if it is not a nanomaterial as defined by ISO [1] and the European Commission [24], a normal approach can be used. Otherwise, it is assumed that it contains 


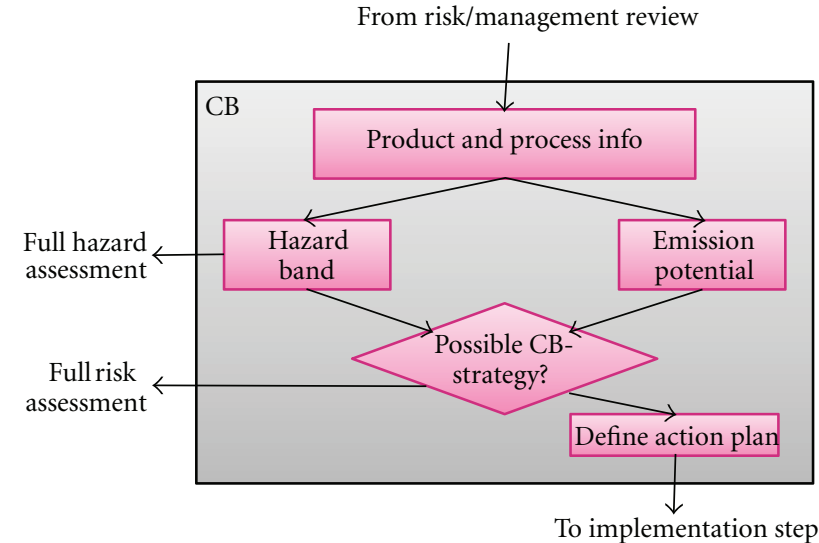

FIGURE 1: The control banding planning step combines hazard and emission potential information to inform about a possible CBstrategy resulting in an action plan, if deemed feasible.

nano-objects that can cross biological barriers [25]. The second step identifies nanomaterials already classified by a relevant authority (e.g., for Nano- $\mathrm{TiO}_{2}$ and Carbon NanoTubes, the Swiss authorities already published recommended exposure limits [8]), in which case that classification should be used for defining the hazard band (see Supplemental Material available online at doi:10.1155/2012/879671). If this (not already classified) nanomaterial is a long biopersistent fibre, then the band is that of the maximum hazard, which requires a full risk assessment. If "no" then the hazard banding process can be applied to this nanoproduct.

When there is a bulk material, an initial allocation of the nanomaterial to one of five hazard bands (HB1-5) relates to the classification of the bulk material according to the CLP Regulation (drawn from COSHH essential tools, reproduced from [22], table in the online supplementary material). In the case where there is no bulk material but there is an analogous material, the process is almost the same, but the level is increased by one band to reflect the added uncertainty. An analogous material is defined in this context to be a substance or material with a similar composition and/or crystalline phase from the same chemical category and with similar documented physicochemical properties (metal oxides, graphite, ceramics, etc.) as the substance of interest [22]. A comprehensive hazard assessment is recommended if there is neither a bulk nor an analogous substance. For the purpose of the $\mathrm{CB}$, the hazard band should be set to level 5 until more information is available. Finally, to account for deficiencies in terms of information on the nanomaterial's toxicity, increment factors are assigned based on the materials' solubility and reactivity. If no information is known about dissolution time or the reactivity, the user should sway to the safe side by increasing the hazard band (or try to find the answer, e.g., from the producer).

Thus, after analysing the product's toxicity criteria and referring to the hazard group allocation of the e-COSHH Essentials tool [26], the hazard levels adopted by the experts who participated in the method's development are presented as follows:

(i) HB1: very low: no significant risk to health;

(ii) HB2: Low: slight hazard—slightly toxic effects rarely requiring medical follow-up;

(iii) HB3: moderate: moderate to significant health effects requiring specific medical follow-up;

(iv) HB4: high: unknown health effects or serious hazard: material highly toxic, sensitising, or with unknown effects on health or the environment. (Emission or exposure in the environment requires a specific survey);

(v) HB5: very high: severe hazard requiring a full hazard assessment by an expert from the central support team.

When duly justified, the hazard band obtained using the given lattice (see Figure 2) can be changed. Such a change should be undertaken only when supported by unequivocal scientific data or by an assessment from a specialist in nanotoxicology.

2.3. Allocation of Emission Potential Bands. Often in control banding hazard bands are combined with exposure bands. However, in our approach, the emission potential is used instead to not depend on current uncertainties related to exposure estimation [20]. The allocation to an emission potential band is defined according to the physical form of the nanomaterial, whether raw or included in a matrix and the process it will endure. The number of workers, the frequency and duration of exposure, the quantity used and existing control measures are not taken into account during the $\mathrm{CB}$ process but need to be considered during the definition of the action plan. The physical form to be considered is that of the material entering the process at the work place evaluated. Four categories of physical forms were defined for the purpose of this approach and are listed below in order of presumed increasing emission potential:

(i) Solid: solid materials containing nanomaterials or having a surface that is nanostructured or covered with nanoparticles.

(ii) Liquid: suspension of free nanoobjects and/or aggregates/agglomerates of nano-objects smaller than $100 \mathrm{~nm}$ in a liquid medium, regardless of its viscosity.

(iii) Powder: mass of nanomaterials (free nanoobjects and/or aggregates/agglomerates of nano-objects smaller than $100 \mathrm{~nm}$ ).

(iv) Aerosol: liquid or solid suspension of nanomaterials (free nano-objects and/or aggregates/agglomerates of nano-objects smaller than $100 \mathrm{~nm}$ ) in a gas (including air).

To account for the natural tendency of certain materials to change from one physical form to another (friable solid yielding a powder, e.g.), emission potential bands are increased accordingly by one or more emission levels. Finally, 


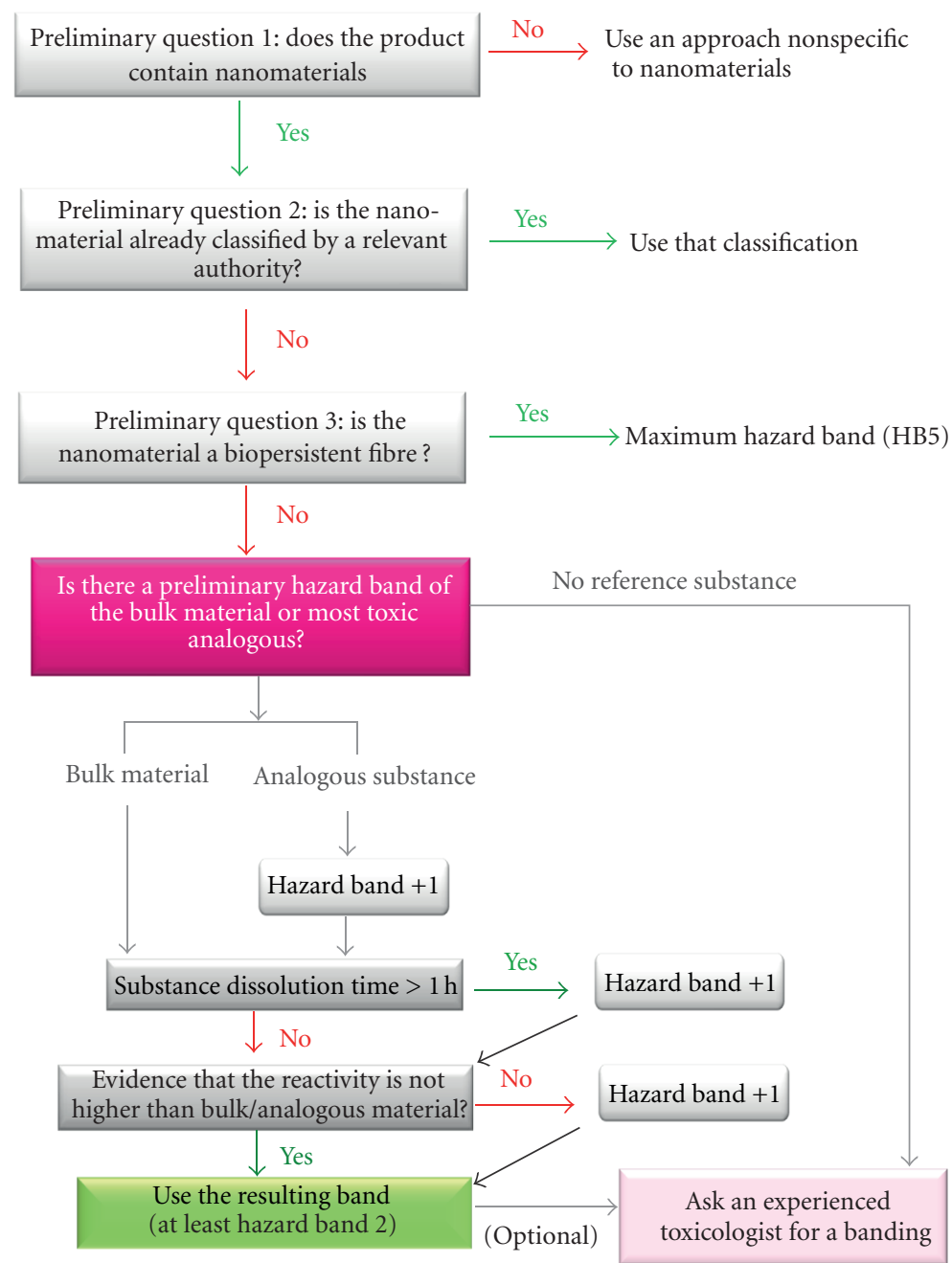

Figure 2: Flow diagram to allocate a MNM to a hazard band according to the level of knowledge on the nanomaterial.

if the physical nature of the material changes as a result of the process used, then an increment in the emission potential band is provided as proposed in Table 1. In a cautious approach, processes generating dust were assumed to lead to aerosols of nanomaterials (i.e., an increase by three bands). If processes are combined then the increments are additive (e.g., melting a solid containing nanomaterials followed by spraying would lead to EP4).

2.4. Allocation of Risk Control Bands. Risk control bands are obtained by overlapping the above-defined hazard bands and emission potential bands. Table 2 shows the matrix of control classes to be implemented with regard to the combination of the hazard level and the emission potential.

Each control band corresponds to technical solutions for collective prevention to be implemented at the work station. They can be distinguished by the level of containment they offer to the user.

(i) CB 1: natural or mechanical general ventilation.

(ii) CB 2: local ventilation: extractor hood, slot hood, arm hood, table hood, and so forth. (iii) $\mathrm{CB}$ 3: enclosed ventilation: ventilated booth, fume hood, closed reactor with regular opening.

(iv) CB 4: full containment: continuously closed systems.

(v) CB 5: full containment and review by a specialist required: seek expert advice.

It should be noted that the wearing of personal protective equipment has deliberately not been taken into account. Indeed, personal protection should be limited to operations for which solutions involving substitution, technical change to a process, or collective protection cannot be implemented.

All materials in hazard band 5 are put into the highest control band. This means that production lines/individual SMEs need to consult with the central support team when they set up a new process with these materials. This will give the central support team an overview on these processes. The added burden on the central support team is considered moderate because in the case of many similar cases present in the company or group of SMEs, the support team will already have collected most of the information necessary for the "full risk assessment." 
TABle 1: Definition of emission potential levels (EP) depending on the physical form of the nanomaterial matrix and specific material transformations for different physical forms.

\begin{tabular}{llcc}
\hline Solid & Liquid & Powder & Aerosol \\
EP1 & EP2 & EP3 & EP4 \\
\hline
\end{tabular}

Specific cases of band modification due to the natural tendency of the material or the matrix

\begin{tabular}{lll} 
Friable solid ${ }^{1}\left(+2\right.$ bands) $\quad$ Highly volatile liquid $^{2}(+1$ band $)$ & $\begin{array}{l}\text { High or moderate } \\
\text { dustiness powder } \\
(+1 \text { band })\end{array}$ \\
\hline
\end{tabular}

Specific cases of band modification due to process operation

Dust generated by external forces ${ }^{4}(+3 \quad$ Powder generated by evaporation

bands) Melting ( +1 band) Dispersion $\quad(+1 /+2$ band according to dustiness of

in liquid ( +1 band)

of $\quad$ Spraying $(+1$ band $)$

${ }^{1}$ Material whose matrix is likely to release particles under low stress [57].

${ }^{2}$ According to INRS ND 2233 [10].

${ }^{3}$ Respirable fraction according to EN 15051 [58].

${ }^{4}$ External forces such as for instance, mechanical forces, electrical forces, lasers, and so forth.

TABle 2: Definition of Control bands (CB) based on hazard bands (HB) and Emission potential bands (EP).

\begin{tabular}{|c|c|c|c|c|c|}
\hline & & \multicolumn{4}{|c|}{ Emission potential bands } \\
\hline & & EP1 & EP2 & EP3 & EP4 \\
\hline \multirow{5}{*}{ Hazard bands } & HB1 & CB 1 & $\mathrm{CB} 1$ & $\mathrm{CB} 2$ & CB 3 \\
\hline & HB2 & CB 1 & CB 1 & $\mathrm{CB} 2$ & $\mathrm{CB} 3$ \\
\hline & HB3 & CB 1 & CB 1 & CB 3 & CB 4 \\
\hline & HB4 & $\mathrm{CB} 2$ & CB 2 & CB 4 & CB 5 \\
\hline & HB5 & $\mathrm{CB} 5$ & $\mathrm{CB} 5$ & CB 5 & CB 5 \\
\hline
\end{tabular}

Based on these control bands, an action plan will be defined that identifies the specific technical and organisational measures that are adapted to the situation of the workplace and the processes. The central support team may decide to provide here additional guidance by recommending for often observed situations equipment and setup procedures; operational conditions; maintenance, control, and cleaning procedures and intervals; access control; documentation and reporting procedures.

The result of the approach presented in this paper is contextual and should be reviewed as soon as circumstances change (process modifications, development of scientific knowledge or state of the art, etc.). In accordance with the principle of continuous improvement, the CB approach deserves to be reiterated in order to update the result.

\section{Rational}

3.1. Rational for the Hazard Banding. The central element of the above-described CB is the classification of materials into levels of increasing hazardousness. This approach corresponds to four key criteria we suggest being central to describe nanomaterial toxicity: the ability to cross biological barriers, the fibrous nature, the biopersistence, and the reactivity of the nanomaterial. These factors were chosen because they can be linked to other physical and chemical properties, such as surface chemistry, crystalline form, particle morphology and size, and so forth $[3,27]$. It is targeting occupational health specialists without detailed expertise in nano-toxicology. A similar, though a bit simpler approach was already successfully used in the Swiss Precautionary Matrix [28].

The Ability to Cross Biological Barriers, was repeatedly reported for nanoparticles. They seem to easily translocate across biological barriers implying that they can carry the toxic substances through membranes [29] into cells and, by translocation [30] to other organs even though only very small quantities do translocate. Thus, this opens the way to novel or unexpected effects in cells or organs. This ability is one key aspects that led to the development of this hazard banding and it is the rational for question 1 and contributes to the question about solubility.

Biopersistence, Combined with the Fibrous Nature, is included because the occupational health literature [3136] suggests that all long respirable and biopersistent fibres should be treated as asbestos unless evidence to the contrary is obtained. This was also suggested from experimental animal testing with carbon nanotubes $[37,38]$. Hence the full hazard assessment is required. Biopersistence in this context can be defined as the ability of a fibre to remain in the lung in spite of the lung's physiological clearance mechanisms. For defining health relevant fibres, existing definitions [39] can be used where fibres have a length over $5 \mu \mathrm{m}$, a diameter less than $3 \mu \mathrm{m}$ and a length-to-diameter ratio over $3: 1$. This definition corresponds to the recently published recommended exposure limit in Switzerland for Carbon-NanoTubes and -NanoFibres [8]. 
Higher Reactivity with regard to the bulk material or analogous material covers different paradigms of the nanomaterials' chemical properties that are relevant for their potential impact on health $[40,41]$. The basic definition of "reactivity" refers to the rate at which a substance tends to undergo a chemical reaction in time. Here we are mostly interested in the surface chemistry and the ability of the material to generate, directly or indirectly, reactive oxygen or nitrogen species [40,42, 43]. It is important in our context because, for instance, a material with a higher specific surface area is expected to have a higher reactivity than a material of the same chemical composition but with a lower specific surface area. The reactivity can also be modified by the inclusion of contaminants that originate from the nanomaterial production processes, which differ from the bulk material. A quantification of the reactivity could be done for example, by calculating the free surface energy [44], by using reactivity assays [45], or by estimating it in comparison to other materials [28]. The hazard band is increased by one band if it is not confirmed that the nanoform of the material has no increased reactivity compared to bulk or analogous material, thus swaying to the cautious side in case of uncertainty.

Solubility refers to the degree to which a material can be dissolved in another material so that a single, homogenous, temporally stable phase results. It is important not to confuse solubility and dispersibility, as we are interested in the potential of a material to lose its particulate character and to change to a molecular or ionic form. This should be stressed, as this distinction may be difficult with colloidal suspensions of nanomaterials. A possible method to assess the solubility of a nanomaterial can be derived from the OECD test guidelines TG105 [46]. Solubility was chosen because of its importance in evaluating biopersistence and biokinetics. An insoluble or poorly soluble nanomaterial will have the opportunity to be translocated in the body from the entry compartment (lungs, gastrointestinal tract, skin, nose) to another and be distributed in the body towards secondary target organs or tissues [25, 47-49]. Accumulation, also at the entry site, enhances the risk of chronic hazardous effects $[33,50]$. The potential hazard of a soluble material will be treated as a toxicology problem of its solutes even though there is a fair potential for differences in toxicity: a finely dispersed soluble nanomaterial will be leading to many spots of high local concentrations around the site of deposition and thus more cells may be exposed to observable effect level, which ultimately would lead to a stronger, organ-wide or systemic effect. While the solubility of a nanomaterial is considered important to assess its hazard level, there is almost no information on the solubility threshold that would be considered to describe a material as highly, moderately or not at all hazardous. We decided to increment the material by one hazard band if it does not completely dissolve in one hour, in water $[25,51]$ or in a simulated lung lining fluid [e.g., in a natural porcine surfactant preparation $[52,53]]$. The limit of one hour is based on evidence that some insoluble nanoparticles may penetrate in the epithelial cells and deeper in lung tissues within one hour of exposure $[29,30,54-56]$, and we focused on a model based on the airways because this is the major route for occupational exposure.

3.2. Rational for the Emission Banding. The emission banding groups the materials into four categories of physical form: solid, liquid, powder, and aerosol. The emission potential increases along this sequence of physical forms. The subsequent band modifications re-allocate situations if the natural tendency of the material or the process operation bring the material into another physical form or a state that in its nature corresponds to that. The following rational was behind the modifications proposed in Table 1:

(i) Friable solids can break up easily into powders [57], thus the banding is increased by two levels, which corresponds to the level of a powder;

(ii) Nanomaterials that are dispersed in a highly volatile liquid [10] may become again powders if the liquid evaporates. To be on the safe side, the band is increased by one level.

(iii) High or moderate dusty powders can easily become airborne [58].

(iv) Generation of dust is—-per definition—a process that creates aerosols.

(v) Melting and dispersion of a solid in a liquid is-per definition - a change of physical form.

(vi) Powder generation is-per definition-a change of physical form. The additional increase in dependence of dustiness was discussed above.

(vii) Spraying is—per definition - the creation of aerosols.

These emission potential bands are based on essential natural concepts and basic definitions and thus are robust and easy to define. It is likely that in some cases, the emission potential band is clearly overestimating the real emission potential. If the resulting control strategy is not easily feasible and costly, the person doing the control banding (e.g., a line manager) can call for a "full risk assessment." In practice this would mean that an expert (e.g., the company's occupational hygienist) evaluates the banding, re-classifies the emission potential and documents this modification in the overarching risk management system.

\section{Outlook}

The here presented CB tool seems easy and simple to apply for managing the risks of individual workplaces since it relies on only a few parameters. However, the CB method applied to manufactured nanomaterials requires assumptions to be formulated on information that is desirable but unavailable. To be able to obtain the necessary parameters for this $\mathrm{CB}$, the user should be proficient in chemical risk prevention and have some basic knowledge on nanomaterials and nanotoxicology. The here proposed approach needs to be embedded in the organization's global risk management system, which comprises many other processes. Thus, the safety and health specialists providing the expertise for this 
system are those that will need to provide also the nanospecific expertise. This approach seems useful not only for industrial companies, but also for academic institutions and professional alliances of small and medium enterprises where the health and safety competencies are provided by external offices.

The proposed $\mathrm{CB}$ approach collapses into a regular CB for nonnanomaterials and for MNM with existing authoritydefined classifications. Thus, it can be integrated into CBbased risk management systems. It was designed to allow for the easy integration of all the expected results from toxicology and risk assessments that are currently being conducted. However, the approach was not yet extensively tested and still needs to be validated to be truly practical for the proposed group of users.

\section{Acknowledgments}

The development of the here proposed control banding approach was financially supported by the French Agency for Food, Environmental, and Occupational Health SafetyANSES. However, it does not necessarily represent the views of ANSES. It was developed by an interdisciplinary and international expert team in request to the French Ministry of Health, in agreement with the Ministries for the Environment and Labour, who had requested that ANSES conduct a collective expert appraisal specifically on Control Banding applied to manufactured nanomaterials.

\section{References}

[1] ISO, ISO/TS 27687, Nanotechnologies: terminology and definitions for nano-objects : nanoparticle, nanofibre and nanoplate2008, Geneva, Switzerland, ISO, 2008.

[2] A. D. Maynard, "Nanotechnology: the next big thing, or much ado about nothing?" Annals of Occupational Hygiene, vol. 51, no. 1, pp. 1-12, 2007.

[3] G. Oberdörster, A. Maynard, K. Donaldson et al., "Principles for characterizing the potential human health effects from exposure to nanomaterials: elements of a screening strategy," Particle and Fibre Toxicology, vol. 2, article 8, 2005.

[4] C. Ostiguy, B. Roberge, C. Woods et al., Engineered Nanoparticles: Current Knowledge about OHS Risks and Prevention Measures, Studies and Research Projects, IRSST, Montréal, Canada, 2nd edition, 2010.

[5] M. Ricaud and O. Witschger, Nanomaterials: Definitions, Toxicological Risk, Characterisation of Occupational Exposure and Prevention Measures, INRS, Paris, France, 2009.

[6] INRS, Valeurs Limites D'exposition Professionnelle aux Agents Chimiques en France, INRS, Paris, France, 2008, 2ème ed2008.

[7] Québec, Regulation respecting occupational health and safety, Editeur officiel du Québec: [S.1], 2011.

[8] Suva, Grenzwerte am Arbeitsplatz 2011 [Occupational Exposure Limits 2011], Suva, Lucerne, Switzerland, 2011.

[9] ACGIH, TLVs and BEIs Based on the Documentation fo the Threshold Limit Values for Chemical Substances and Physical Angents \& Biological Exposure Indices, ACGIH. XVII, Cincinnati, Ohio, USA, 2010.

[10] R. Vincent, F. Bonthoux, G. Mallet et al., "Méthodologie d'évaluation simplifiée du risque chimique: un outil d'aide à la décision," Hygiène et Sécurité du Travail, vol. 200, pp. 39-62, 2005.

[11] A. D. Maynard, R. J. Aitken, T. Butz et al., "Safe handling of nanotechnology," Nature, vol. 444, no. 7117, pp. 267-269, 2006.

[12] S. Y. Paik, D. M. Zalk, and P. Swuste, "Application of a pilot control banding tool for risk level assessment and control of nanoparticle exposures," Annals of Occupational Hygiene, vol. 52, no. 6, pp. 419-428, 2008.

[13] NIOSH, Progress Toward Safe Nanotechnology in the Workplace: A Report from the NIOSH Nanotechnology Research Center, NIOSH, Cincinnati, Ohio, USA, 2007.

[14] B. D. Naumann, E. V. Sargent, B. S. Starkman, W. J. Fraser, G. T. Becker, and G. D. Kirk, "Performance-based exposure control limits for pharmaceutical active ingredients," American Industrial Hygiene Association Journal, vol. 57, no. 1, pp. 33-42, 1996.

[15] I. M. Brooke, "A UK scheme to help small firms control health risks from chemicals: toxicological considerations," Annals of Occupational Hygiene, vol. 42, no. 6, pp. 377-390, 1998.

[16] A. N. I. Garrod, P. G. Evans, and C. W. Davy, "Risk management measures for chemicals: the "COSHH essentials" approach," Journal of Exposure Science and Environmental Epidemiology, vol. 17, supplement 1, pp. S48-S54, 2007.

[17] H. Marquart, H. Heussen, M. Le Feber et al., "'Stoffenmanager', a web-based control banding tool using an exposure process model," Annals of Occupational Hygiene, vol. 52, no. 6, pp. 429-441, 2008.

[18] R. M. Russell, S. C. Maidment, I. Brooke, and M. D. Topping, "An introduction to a UK scheme to help small firms control health risks from chemicals," Annals of Occupational Hygiene, vol. 42, no. 6, pp. 367-376, 1998.

[19] BAuA, "Easy-to-use workplace control scheme for hazardous substances: a practical guide for the application of the German hazardous substance ordinance by small and medium-sized enterprises working with hazardous substances without workplace limit values," Dortmund, Germany, BAuA, Annexes, 2006.

[20] G. Hunt and M. Riediker, "Building expert consensus on problems of uncertainty and complexity in nanomaterials safety," Nanotechnology Perceptions, vol. 7, no. 2, pp. 82-98, 2011.

[21] D. M Zalk, R. Kamerzell, S. Paik, J. Kapp, D. Harrington, and P. Swuste, "Risk level based management system: a control banding model for occupational health and safety risk management in a highly regulated environment," Industrial Health, vol. 48, no. 1 , pp. 18-28, 2010.

[22] C. Ostiguy, R. Michael, T. Jerome et al., "Development of a specific control banding tool for nanomaterials: report," Tech. Rep., Afsset, Maisons-Alfort, France, 2010.

[23] BSI, BS OHSAS 18001:2007. Occupational Health and Safety Management Systems - Requirements, BSI, London, UK, 2007.

[24] EC, "Commission Recommendation of 18 October 2011 on the definition of nanomaterial," Official Journal of the European Union, vol. 275, no. 54, pp. 38-40, 2011.

[25] G. Oberdörster, Z. Sharp, V. Atudorei et al., "Extrapulmonary translocation of ultrafine carbon particles following wholebody inhalation exposure of rats," Journal of Toxicology and Environmental Health A, vol. 65, no. 20, pp. 1531-1543, 2002.

[26] HSE, Control of substances hazardous to health: the control of substances hazardous to health regulations 2002 (as amended): approved code of practice and guidance. 5th ed, Sudbury, UK, HSE Books, 2005. 
[27] H. Bouwmeester, I. Lynch, H. J. P. Marvin et al., "Minimal analytical characterization of engineered nanomaterials needed for hazard assessment in biological matrices," Nanotoxicology, vol. 5, no. 1, pp. 1-11, 2011.

[28] J. Höck, H. Heinrich, H. Karl et al., "Precautionary Matrix for Synthetic Nanomaterials," Berne, Switzerland, Swiss Federal Office of Public Health and Federal Office for the Environment, 2008.

[29] M. Geiser, B. Rothen-Rutishauser, N. Kapp et al., "Ultrafine particles cross cellular membranes by nonphagocytic mechanisms in lungs and in cultured cells," Environmental Health Perspectives, vol. 113, no. 11, pp. 1555-1560, 2005.

[30] W. G. Kreyling, M. Semmler, F. Erbe et al., "Translocation of ultrafine insoluble iridium particles from lung epithelium to extrapulmonary organs is size dependent but very low," Journal of Toxicology and Environmental Health A, vol. 65, no. 20, pp. 1513-1530, 2002.

[31] K. Donaldson and C. L. Tran, "An introduction to the shortterm toxicology of respirable industrial fibres," Mutation Research, vol. 553, no. 1-2, pp. 5-9, 2004.

[32] K. Donaldson, R. Aitken, L. Tran et al., "Carbon nanotubes: a review of their properties in relation to pulmonary toxicology and workplace safety," Toxicological Sciences, vol. 92, no. 1, pp. 5-22, 2006.

[33] K. Donaldson, F. A. Murphy, R. Duffin, and C. A. Poland, "Asbestos, carbon nanotubes and the pleural mesothelium: a review of the hypothesis regarding the role of long fibre retention in the parietal pleura, inflammation and mesothelioma," Particle and Fibre Toxicology, vol. 7, article 5, 2010.

[34] T. W. Hesterberg, W. C. Miiller, R. Mast, E. E. McConnell, D. M. Bernstein, and R. Anderson, "Relationship between lung biopersistence and biological effects of man-made vitreous fibers after chronic inhalation in rats," Environmental Health Perspectives, vol. 102, no. 5, pp. 133-137, 1994.

[35] A. B. Kane, "Epidemiology and pathology of asbestos-related diseases," Reviews in Mineralogy, vol. 28, no. 1, pp. 347-359, 1993.

[36] A. Searl, D. Buchanan, R. T. Cullen, A. D. Jones, B. G. Miller, and C. A. Soutar, "Biopersistence and durability of nine mineral fibre types in rat lungs over 12 months," Annals of Occupational Hygiene, vol. 43, no. 3, pp. 143-153, 1999.

[37] C. A. Poland, R. Duffin, I. Kinloch et al., "Carbon nanotubes introduced into the abdominal cavity of mice show asbestoslike pathogenicity in a pilot study," Nature Nanotechnology, vol. 3, no. 7, pp. 423-428, 2008.

[38] A. Takagi, A. Hirose, T. Nishimura et al., "Induction of mesothelioma in p53+/- mouse by intraperitoneal application of multi-wall carbon nanotube," Journal of Toxicological Sciences, vol. 33, no. 1, pp. 105-116, 2008.

[39] WHO/EURO, Reference methods for measuring airborne man-made mineral fibres (MMMF), Environmental health, ed. W.E.T.C.f.M.a.E.A. MMMF, Copenhagen: WHO Regional Office for Europe, VII, 55, 1985.

[40] D. M. Brown, M. R. Wilson, W. MacNee, V. Stone, and K. Donaldson, "Size-dependent proinflammatory effects of ultrafine polystyrene particles: a role for surface area and oxidative stress in the enhanced activity of ultrafines," Toxicology and Applied Pharmacology, vol. 175, no. 3, pp. 191-199, 2001.

[41] K. Donaldson, X. Y. Li, and W. MacNee, "Ultrafine (nanometre) particle mediated lung injury," Journal of Aerosol Science, vol. 29, no. 5-6, pp. 553-560, 1998.

[42] D. M. Brown, V. Stone, P. Findlay, W. MacNee, and K. Donaldson, "Increased inflammation and intracellular calcium caused by ultrafine carbon black is independent of transition metals or other soluble components," Occupational and Environmental Medicine, vol. 57, no. 10, pp. 685-691, 2000.

[43] E. Oberdörster, "Manufactured nanomaterials (fullerenes, C60) induce oxidative stress in the brain of juvenile largemouth bass," Environmental Health Perspectives, vol. 112, no. 10, pp. 1058-1062, 2004.

[44] J.-P. Jolivet, C. Froidefond, A. Pottier et al., "Size tailoring of oxide nanoparticles by precipitation in aqueous medium. A semi-quantitative modelling," Journal of Materials Chemistry, vol. 14, no. 21, pp. 3281-3288, 2004.

[45] J.-J. Sauvain, S. Deslarzes, and M. Riediker, "Nanoparticle reactivity toward dithiothreitol," Nanotoxicology, vol. 2, no. 3, pp. 121-129, 2008.

[46] OECD, Water Solubility, TG 105, in OECD Guideline for the Testing of Chemicals. Section 1, Physical-Chemical Properties, OECD, Paris, France, 1995.

[47] J. Chen, M. Tan, A. Nemmar et al., "Quantification of extrapulmonary translocation of intratracheal-instilled particles in vivo in rats: effect of lipopolysaccharide," Toxicology, vol. 222, no. 3, pp. 195-201, 2006.

[48] A. Nemmar, P. H. M. Hoet, and B. Nemery, "Translocation of ultrafine particles," Environmental Health Perspectives, vol. 114, no. 4, pp. A211-A212, 2006.

[49] G. Oberdörster, Z. Sharp, V. Atudorei et al., "Translocation of inhaled ultrafine particles to the brain," Inhalation Toxicology, vol. 16, no. 6-7, pp. 437-445, 2004.

[50] G. Oberdörster, "Significance of particle parameters in the evaluation of exposure-dose-response relationships of inhaled particles," Inhalation Toxicology, vol. 8, supplement, pp. 73-89, 1996.

[51] S. Takenaka, E. Karg, W. Kreyling et al., "Distribution pattern of inhaled ultrafine gold particles in the rat lung," Inhalation Toxicology, vol. 18, no. 10, pp. 733-740, 2006.

[52] C. Schleh and J. M. Hohlfeld, "Interaction of nanoparticles with the pulmonary surfactant system," Inhalation Toxicology, vol. 21, no. 1, pp. 97-103, 2009.

[53] C. Schleh, C. Mühlfeld, K. Pulskamp et al., "The effect of titanium dioxide nanoparticles on pulmonary surfactant function and ultrastructure," Respiratory Research, vol. 10, article 90, 2009.

[54] M. Geiser and W. G. Kreyling, "Deposition and biokinetics of inhaled nanoparticles," Particle and Fibre Toxicology, vol. 7, article 2, 2010.

[55] M. Semmler, J. Seitz, F. Erbe et al., "Long-term clearance kinetics of inhaled ultrafine insoluble iridium particles from the rat lung, including transient translocation into secondary organs," Inhalation Toxicology, vol. 16, no. 6-7, pp. 453-459, 2004.

[56] M. Semmler-Behnke, S. Takenaka, S. Fertsch et al., "Efficient elimination of inhaled nanoparticles from the alveolar region: evidence for interstitial uptake and subsequent reentrainment onto airways epithelium," Environmental Health Perspectives, vol. 115, no. 5, pp. 728-733, 2007.

[57] S. F. Hansen, E. S. Michelson, A. Kamper, P. Borling, F. StuerLauridsen, and A. Baun, "Categorization framework to aid exposure assessment of nanomaterials in consumer products," Ecotoxicology, vol. 17, no. 5, pp. 438-447, 2008.

[58] BSI, BS EN 15051:2006, Workplace Atmospheres: Measurement of the Dustiness of Bulk Materials: Requirements and Reference Test Methods, BSI, London, UK, 2006. 

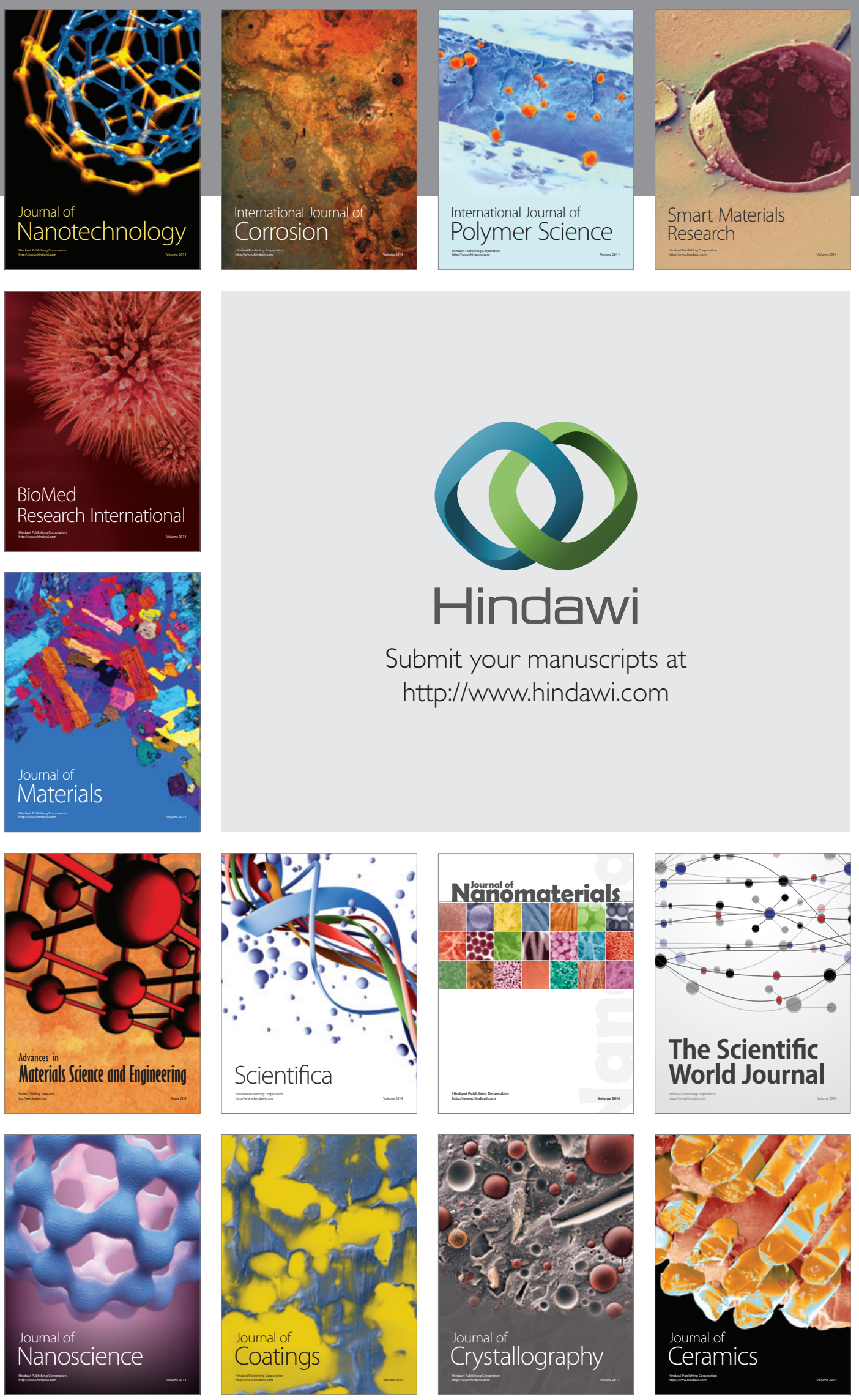

The Scientific World Journal

Submit your manuscripts at

http://www.hindawi.com

\section{World Journal}

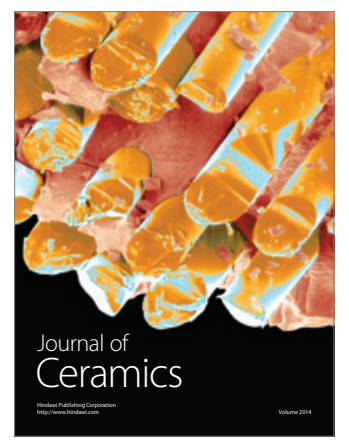

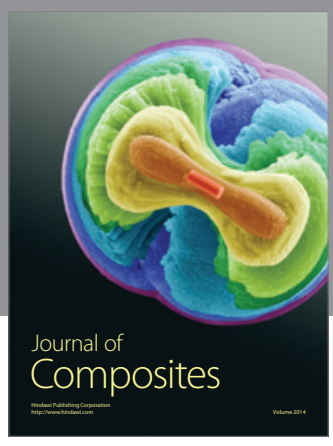
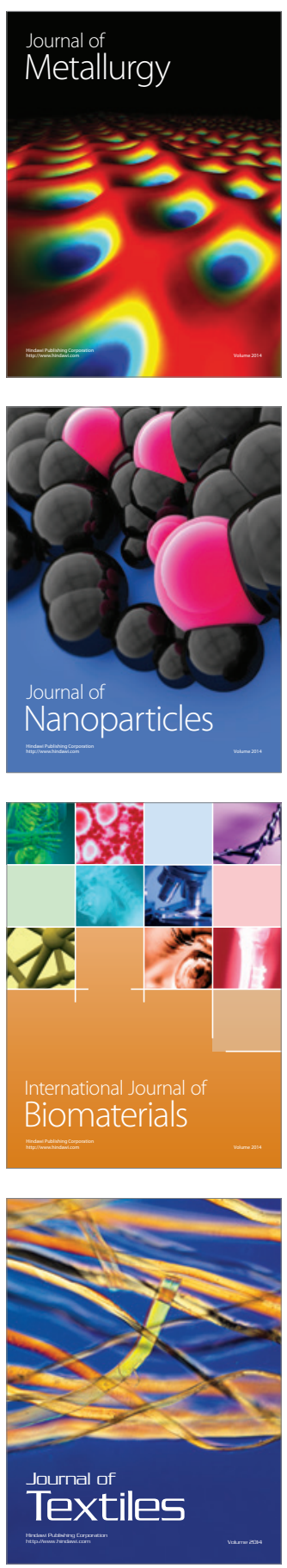